E3S Web of Conferences 1, 32008 (2013)

DOI: $10.1051 / \mathrm{e} 3$ sconf $/ 20130132008$

(C) Owned by the authors, published by EDP Sciences, 2013

\title{
Zero Valent Iron Nanoparticle Assisted Electrocoagulation of Arsenic with electromagnetic Separation of Solids
}

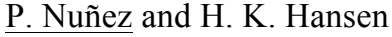 \\ Departamento de Ingeniería Química y Ambiental, Universidad Técnica Federico Santa María, Avenida España 1680, \\ Valparaíso, CHILE, henrik.hansen@usm.cl
}

\begin{abstract}
A new arsenic removal process was designed combining: 1) iron nanoparticle addition, b) electrocoagulation, and c) electromagnetic separation. Results showed that arsenic could be removed with more than $99 \%$ efficiency from liquid waste samples. Parameters that were found to have importance on the process were: a) nanoparticle dosage, b) electric voltage drop during electrocoagulation, b) $\mathrm{pH}$ of the solution, d) arsenic concentration, and e) electromagnetic field distribution during solid separation. Arsenic could efficiently be removed by iron nanoparticles during electrocoagulation. Afterwards the arsenic containing particles were separated from the solution by electromagnetic fields. This new process could be a feasible alternative to conventional arsenic treatment in liquid waste streams.
\end{abstract}

Key words: Arsenic, Iron nanoparticles, electrocoagulation, magnetic separation.

\section{Introduction}

The mining industry in Chile is one of the largest consumers of water after the food industry. Codelco Norte Division alone uses a volume of more than 200 liters per second of water to supply their production processes in this area. At the same time the water is becoming a commodity so the water use must be optimized and as much of it as possible to be recycled in the industrial processes. A major problem of mining effluents is that these waters have high concentration of heavy metals and arsenic. In order to recirculate the water this should be treated by removing heavy metals, when increasing the $\mathrm{pH}$ from acidic to neutral.

Currently, the removal of heavy metals and arsenic is one of the biggest problems in the industry and still a highly efficient process for removing arsenic is missing. The usual treatment consists of either a ferric treatment or a sacrifice electrocoagulation with iron electrodes.

A new method, where electrocoagulation is combined with a zero valent iron nanoparticle addition, has shown great potential. One drawback with this combined treatment is the slow settling of the nanoparticles, which would require very large clarifiers in industrial plants. A possible improvement could be the introduction of an electromagnetic separator (EMS) using the magnetic properties of the nanoparticles.

\section{Materials and Methods}

The total proposed arsenic removal process consists in three parts as indicated on Figure 1. First step is an electrocoagulation unit with nanoparticle addition, where the objective is to adsorb/precipitate arsenic from the acid wastewater. Second step is a clarification unit with the aim to concentrate the solids and have a flow of treated wastewater. The third step is an EMS unit, where the magnetic iron nanoparticles with arsenic are removed from the liquid phase towards magnets.

The electrocoagulation process used current densities of around $150 \mathrm{~A} / \mathrm{m}^{2}$ for treating the wastewater batch wise. This treated wastewater was added in a continuous way to the clarifier and subsequently to the EMS unit. Iron nanoparticles were acquired in the Czech Republic at the company Nanoiron. In general, the main objective with this present work is the efficiency of the EMS and not the electrocoagulation or clarifier units. The efficiency of the EMS unit was evaluated as the outlet concentration of solids as a fraction of the inlet concentration. Typical inlet concentrations was around $1.7 \mathrm{mg} \mathrm{L}^{-1}$. 


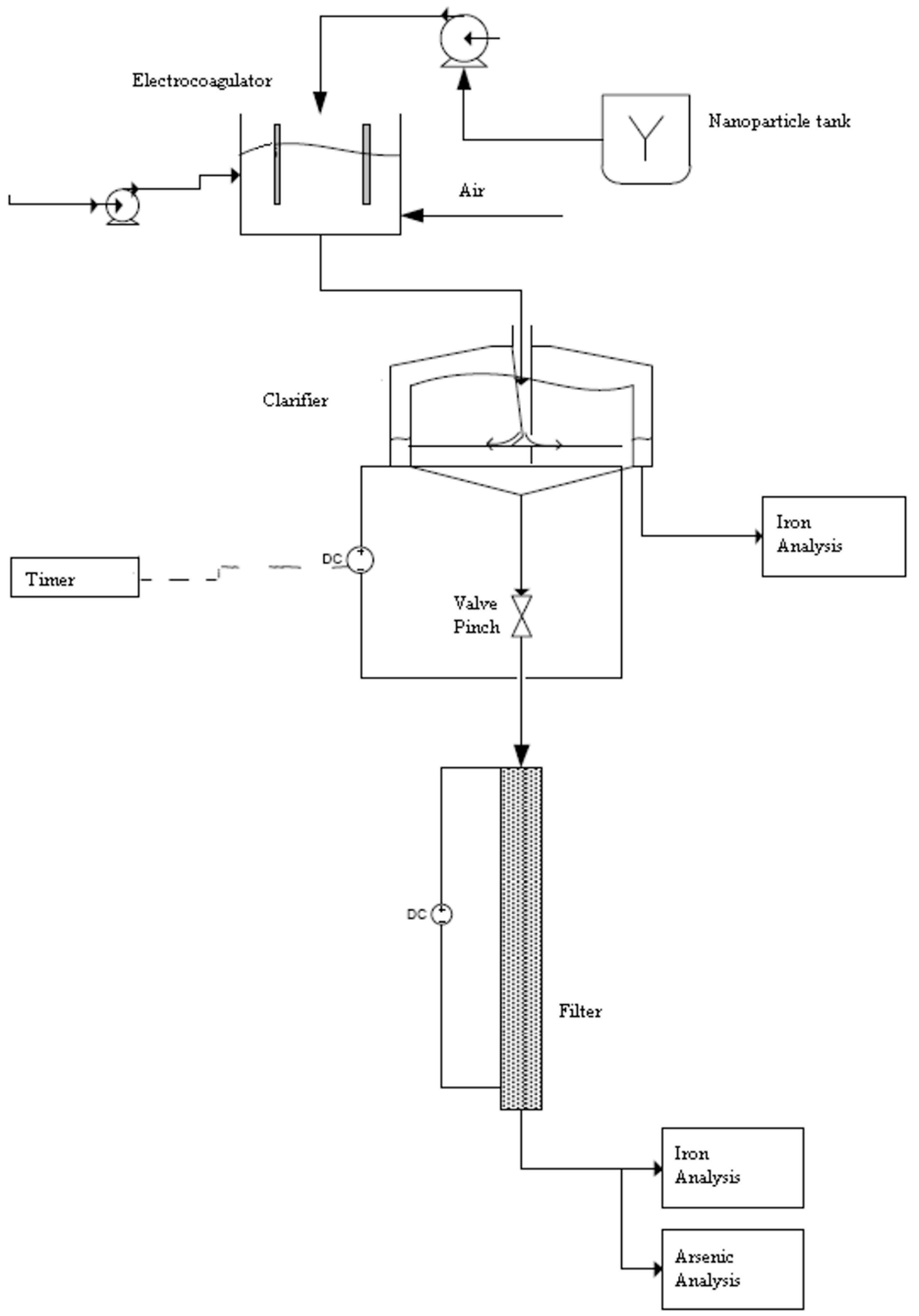

Fig. 1. Total process diagram including electrocoagulation, clarifier and electromagnetic separation (filter) units. 


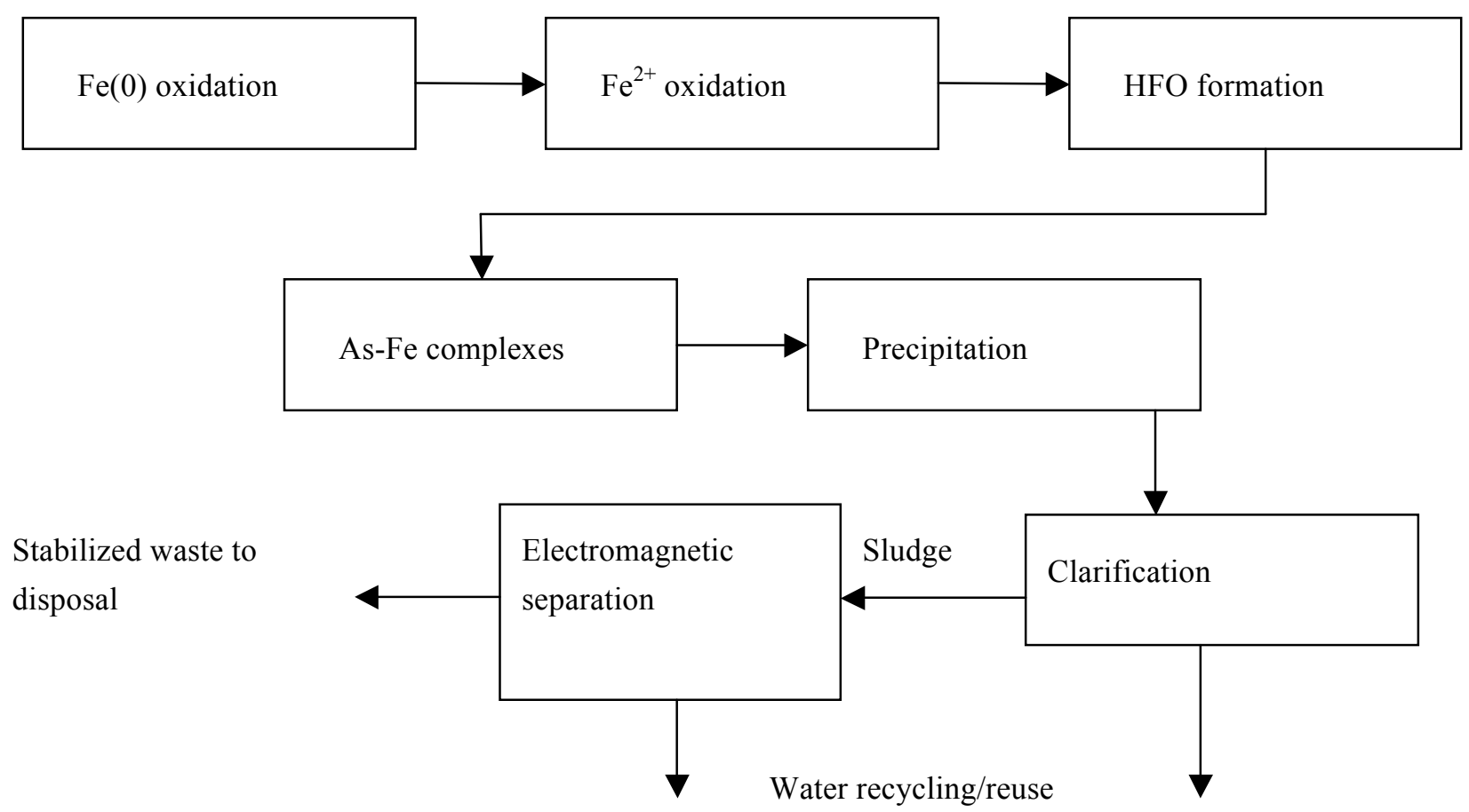

Fig. 2. Suggested reaction pathways.

\section{Results and Discussion}

According to the experimental results it can be concluded that the electrocoagulation process using nZVI is quite efficient in terms of arsenic removal from solutions such as mining wastewaters. A high percentage of pollutant removal close to $99 \%$ was obtained. Optimal conditions were found operating in variables such as $\mathrm{pH}$, time of removal and current density.

It was found that arsenic removal was better at acid $\mathrm{pH}$ (around 2.5) than at neutral $\mathrm{pH}$. This is important since copper smelter wastewater normally is acidic, and no neutralization would be necessary before the arsenic treatment.

One uncertainty with the arsenic removal efficiency could be the structure of nZVI. To possess a "Core Shell" structure, the outer film of nZVI can cause failure to achieve $\mathrm{Fe}^{+2}$ oxidation so the reaction processes after this stage would not be achieved. In other words, the nanoparticles are very stable and one should use a more effective oxidizing agent at acidic $\mathrm{pH}$ to achieve nZVI oxidation.

A first-order kinetic model for arsenic removal was established. It should be noted that this model is basic and is a first attempt to model the experimental phenomena that occur with the nanoparticles and arsenic. The general scheme for the arsenic removal by the combined electrocoagulation/nanoparticle treatment including the formation of hydrated ferric oxides (HFO) can be summarized in Figure 2. Also included in the figure is the electromagnetic separation of the arsenic containing iron nanoparticles, which have magnetic properties. The electromagnetic field could be varied by the electric current and higher solid removal efficiencies could be obtained.

Regarding the electromegantic separation, the results showed that the electromagnetic filtration of iron nanoparticles is possible and is able to remove the major part (more than 60-70\%) of the nanoparticles contained in the treated wastewater suspension. The process has been applied even at a very low residence time (1-3 s), and still it was possible to remove the majority of the solids.

In this work, the lab setup only treated relatively small flows of wastewater. Therefore, for future research it is of great importance to have a larger amount of wastewater with nanoparticles and furthermore also create a way to increase the residence time within the filter at least a 5 times.

\section{Conclusion}

Arsenic could efficiently be removed by iron nanoparticles during electrocoagulation. Afterwards the arsenic containing particles were separated from the solution by electromagnetic fields. This new process could be a feasible alternative to conventional arsenic treatment in liquid waste streams.

\section{Acknowledgements}

The authors thank the financial supports providing from Chilean FONDECYT Project N| 1120111. 